\title{
Vivienda compartida y arrimados en la zona metropolitana de la ciudad de México
}

Judith Villavicencio

UAM-A

INTRODUCCIÓN

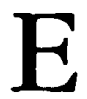

n México, como en la mayor parte de los países de América Latina, la década de los ochenta fue un periodo de profundo deterioro de las condiciones de vida para gran parte de la población y como resultado de la crisis que afectó a las economías de la región. Un fenómeno estrechamente relacionado con ese deterioro y con la crisis en general fue el crecimiento de la llamada "vivienda popular", es decir el aumento de población de las ciudades (espe. cialmente de las grandes ciudades del país) que no pudo económicamente obtener vivienda en el mercado formal o a través de la acción del Estado, teniendo que recurrir a soluciones habitacionales propias.

En la ciudad de México, una solución popular importante continuó siendo la vivienda autoconstruida en la periferia, especialmente hacia el norte y poniente de la ciudad, en áreas pertenecientes a los municipios conurbados del estado de México. Sin embargo, en el periodo señalado se han podido observar también formas diferentes de solución en vivienda que han significado, más que una expansión periférica, una redensificación de áreas habitacionales incor- 
poradas con anterioridad a la mancha urbana.

Las causas de estas pautas de poblamiento diferente han sido señaladas por algunos estudios ${ }^{1}$ relacionando dicho esquema con el deterioro económico de la población y la disminución de la oferta de suelo barato (que han caracterizado a la ciudad de México en los últimos años), que hacen cada vez más difícil para los sectores populares el transformarse en propietarios autoconstructores de nuevas viviendas.

En líneas generales, el proceso de redensificación ha caracterizado, principalmente, a colonias populares que tiempo atrás fueron periféricas y que actualmente están ya consolidadas y tienen el equipamiento y los servicios principales. Espacialmente, esta redensificación se ha efectuado mediante nuevas construcciones en los espacios libres de los predios o a través de la anexión de niveles superiores (uno o dos pisos) a la vivienda original. Estas construcciones o nuevos espacios de vivienda se han destinado, normalmente, al alquiler o a ser compartidos por otra familia.

El alquiler popular representa, en la actualidad, una modalidad importante de acceso a la vivienda para los sectores de bajos ingresos y ha comenzado a ser estudiada con mayor profundidad en México. El compartir es, en cambio, una modalidad de acceso a la vivienda poco investigada y de la cual existe un conocimiento muy limitado en relación con la población que com. parte y el proceso de compartir.

I Coulomb, Inquilinato, 1990; Gilbert, "Arrendatarios", 1987.
Por lo anterior, este trabajo pretende contribuir al conocimiento de la vivienda compartida y de sus ocupantes, los "arrimados" de la ciudad de México, con el doble objetivo de profundizar en el estudio de una de las formas en que se manifiesta el proceso de urbanización en la actualidad, y de avanzar en el análisis de las condiciones de vida de una parte de la población de las grandes ciudades.

\section{LA VIVIENDA COMPARTIDA. \\ PlaNTEAMIENTO DEl PROBlema}

El compartir es una forma de tener acceso a la vivienda y que permite a una familia utilizar toda o parte de una casa que pertenece a otro (propietario o inquilino) y en la cual también reside el teniente oficial. El compartir no es el resultado de un trato de tipo comercial; es, más bien, un acuerdo de carácter solidario entre familias que parece beneficiar, preferentemente, al que demanda compartir ("arrimado") $y$, sin duda, representa una alternativa para aquellos que no pueden (o no quieren) transformarse en propietarios o alquilar una vivienda.

El compartir la vivienda no es, en sí, un fenómeno nuevo en México y ha constituido una fórmula habitual y transitoria para iniciar la vida familiar de muchas parejas recién conformadas, especialmente entre los sectores populares. Lo que sí parece novedoso es la persistencia alcanzada por la categoría de "arrimado" en los últimos años, la magnitud que aparentemente ha adquirido el fenómeno de compartir una vivienda y las crecientes dificul- 
tades de la población que comparte, para modificar esta forma de tenencia $\mathrm{y}$ acceder a una vivienda individual.

Por lo tanto, es necesario estudiar la vivienda compartida profundizando, en primer término, en las especificidades del proceso y sus causas. Investigar, por ejemplo, las razones que llevan a una familia a compartir (y no a alquilar o a comprar) una vivienda; cómo se definen y cuáles son las con. diciones que se establecen para compartir y qué tan estable y persistente es esta forma de ocupación.

Del mismo modo, se requiere avanzar en el conocimiento de la población involucrada en el proceso: tamaño de la familia "arrimada", edad de los jefes y de los hijos, relación con la familia que los cobija, actividades económicas que desarrollan, ingresos, etc. En este sentido es muy importante detectar si es una solución sólo de los sectores populares o si otros grupos socioeconómicos han recurrido a este tipo de solución habitacional para poder establecer así una relación entre este fenómeno, la crisis y el deterioro económico de la población. ${ }^{2}$

Por último, es necesario investigar las condiciones materiales en que viven los "arrimados" y las características de las viviendas que ocupan: tamaño de la vivienda, dotación de servicios,

\footnotetext{
2 En algunos países de América Latina como Argentina, Chile y Uruguay, parte importante de los sectores medios de la población, que por lo general habian tenido acceso a viviendas individuales, debió recurrir a la vivienda compartida como solución habicacional durante los ochenta. Este fenómeno tuvo directa relación con el deterioro económico de esos sectores en el periodo.
}

etc., así como su localización a nivel interurbano. Esto último, con la intención de detectar si es un fenómeno propio de las áreas populares redensificadas o se presenta también en otros tipos de vivienda en el interior de la ciudad (céntricas deterioradas, conjuntos habitacionales, etcétera).

\section{COMPARTIR IA VIVIENDA. ALGUNAS características básicas}

Algunas de las interrogantes anteriores han tratado de ser respondidas a través de un estudio de campo efectuado entre julio y octubre de $1988 .{ }^{3}$ Como punto de partida se plantearon algunas hipótesis generales que es necesario señalar dados los resultados obtenidos.

Así, se pensaba que quienes llegaban a compartir una vivienda eran, principalmente, familiares muy cercanos de quienes la detentaban oficialmente (generalmente propietarios); y que constituían parejas muy jóvenes, recién formadas o eran solteros y, en muchos casos, migrantes recientes a la ciudad. Además, a la situación de compartir se la definía como temporal, pues el deseo de los "arrimados" era transformase rápidamente en propictarios o inquilinos independientes y buscaban constantemente oportunidades de hacerlo. Por último, se plan-

3 La investigación de campo formo parte de un estudio sobre vivienda de alquiler, desarrollado por el Centro de la Vivienda (CENv1) para el IDRC entre 1988 y 1990 . Se agradece a CENV haber proporcionado los antecedentes estadísticos que han hecho posible el desarrollo de este trabajo. 
teaba también que la vivienda se compartía principalmente por problemas económicos y que, por lo tanto, en México sería una solución adoptada sólo por grupos con dificultades específicas en este sentido.

Como punto de partida y a efectos del trabajo de campo (encuesta a los hogares) se definió como arrimado a: "una familia nuclear o extensa que comparte una casa o un predio con otra familia a la cual pertenece la tenencia oficial de la vivienda (propiedad o alquiler), sin mediar un acuerdo comercial entre ambas".

Se consideró como "familia nuclerar o extensa" separada de la familia principal (es decir, que comparte), a aquella que cocinaba y comía, por lo general, en forma independiente. Como familia nuclear se incluyó también a las personas solas.

Si bien esta definición inicial parecía permitir la identificación de los hogares a encuestar, el estudio se enfrentó a ciertas dificultades a nivel del trabajo empírico porque se presentaron las siguientes situaciones muy comunes: $a$ ) problemas para distinguir a los "arrimados" en las viviendas habitadas por familias extensas, constituidas por padres, hijos, nietos, etcétera $;{ }^{4} b$ )

4 Es bastante común, entre los sectores populares, que el hijo varón que constituye una nueva familia lleve a la mujer a vivir en la casa de sus padres, al menos por un tiempo. En un sentido estricto, este nuevo núcleo debe ser conceptualizado como arrimado pero, al mismo tiempo, es el resultado de ciertas pautas culturales que llevan a considerar natural el procedimiento $y$, en muchos casos, los padres se preparan con anticipación para ello (construyen nuevos cuartos en la vivienda, etc.). De ahí que familias arrimadas que podían diferenciarse perfectamente bien de las que detentaban la vivienda y, sin embargo, no siempre separaban sus actividades de cocinar y comer $o$, incluso, su gasto en alimentación; $c$ ) familias que aparecían como arrimadas y que eran, en realidad, inquilinos de la familia principal, la cual no quería admitirlo por temor a acciones oficiales (cobros de impuestos) o a intentos de usurpación de la propiedad por extraños ya que, en algunos casos, no estaba todavía registrada oficialmente (carencia de escrituras, etc.); d) "arrimados" que se confundían con inquilinos informales, dado que habían adquirido compromisos materiales o económicos con la familia que los cobijaba, de manera similar a los que se establecen en ciertos acuerdos de alquiler (pago de predial, pago de servicios, tareas domésticas, etcétera).

Superadas estas indefiniciones (por lo general con ayuda de los propios entrevistados o personas que conocían bien a la población objetivo) se identificaron 80 casos en dos colonias populares de la zona metropolitana de la ciudad de México: Santo Domingo y Fi Sol. La primera de ellas está localizada en la delegación de Coyoacán en el Distrito Federal y se estableció en esa zona a partir de una invasión de terrenos comunales efectuada en 1972 siendo regularizada posteriormente. La colonia El Sol se ubica en el municipio de Nezahualcóyotl, en el estado de México, y se desarrolló mediante la acción de fraccionadores clandestinos que comenzaron a vender terreel arrimado no siempre sea identificado como tal en el interior de la familia. 


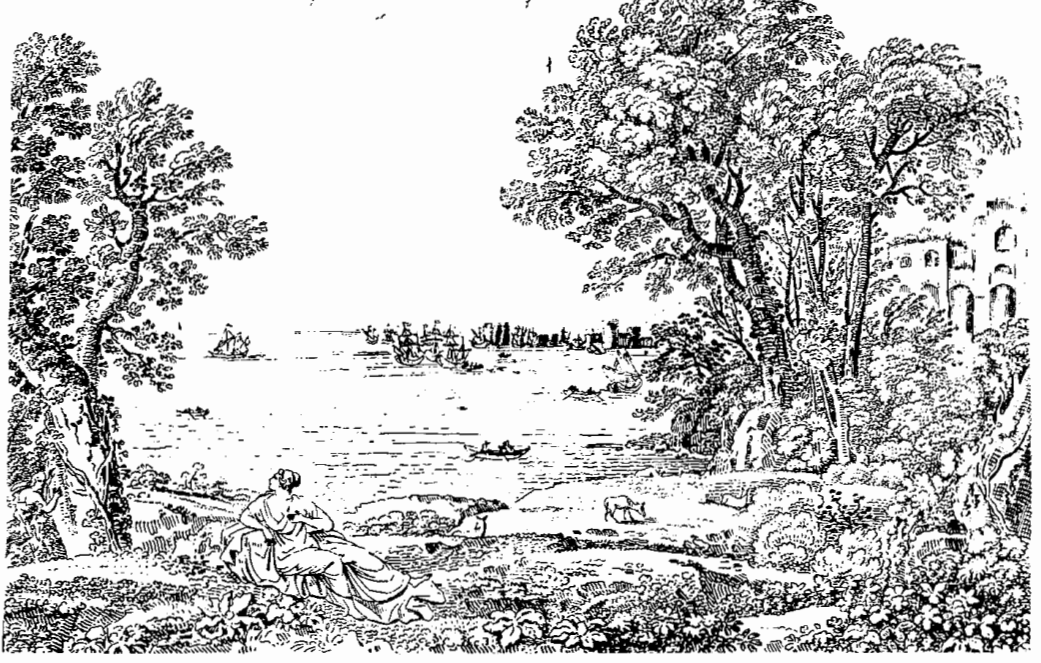

nos ilegalmente en los años sesenta y que también fue postcriormentc regularizada.

Ambas colonias desarrollaron sus servicios, equipamiento y viviendas con el trabajo de los colonos y en la actualidad, con más de 20 años de establecidas, están incorporaclas perfectamente a la mancha urbana y pueden ser consideradas dentro del tipo de colonias populares consolidadas.

Los resultados principales del estudio se refieren $\mathbf{a}$ :

LA POBLACIÓN QUE COMPARTE. LOS "ARRIMADOS"

\section{a) Comportamiento demográfico}

Al contrario de lo que se esperaba, las familias arrimadas no son especial- mente jóvenes. En El Sol, 25\% de los varones jefes de hogar son menores de 25 años y 49\%, menores de 30; pero $37.5 \%$ tiene entre 30 y 50 años.

Del mismo modo, no se caracterizan por tener sólo hijos pequeños o no tener hijos. Si bien son más los hogares con hijos entre 0 y 4 años (en $50 \%$ de los hogares de El Sol y en $\mathbf{2 . 5 \%}$ de los de Santo Domingo hay hijos de esta edad), son también numerosas las familias arrimadas que tienen hijos de 15 años o más ( $25 \%$ en El Sol y $22 \%$ en Santo Domingo).

En cuanto al tipo y tamaño de la familia, la gran mayoría son hogares nucleares $(90 \%$ en El Sol y $\mathbf{8 2 . 5 \%}$ en Santo Domingo) configurados por familias pequeñas (35\% de los hogares en El Sol y 37.5\% en Santo Domingo se componen de una a tres personas) 
y también grandes (en ambas colonias, el $32.5 \%$ de los hogares son de cinco o más personas).

Finalmente, llama la atención que la gran mayoría de los arrimados (52.5\% en El Sol y $60 \%$ en Santo Domingo) son originarios del área metropolitana de la ciudad de México, por lo que se los considera no migrantes. Esto constituye una diferencia importante entre ellos y otros tipos de habitantes de las colonias encuestadas (en la colonia El Sol, sólo $28 \%$ de los propietarios y $23.8 \%$ de los inquilinos son no migrantes y en Santo Domingo lo son $47.5 \%$ y $40 \%$ respectivamente).

\section{b) Características socioeconómicas}

En la fecha de la encuesta, $92.8 \%$ de los jefes varones (en ambas colonias) declaró tener un trabajo remuneracto; $1.47 \%$ estaba clesempleado; $1.4 \%$ era estudiante y $1.4 \%$ estaba jubilado o pensionado. 5 De los que trabajan, la mayor parte se insertaba en el sector servicios $(30 \%$ en $\mathrm{E} 1$ Sol y $37.5 \%$ en Santo Domingo) y tenía categorías muy variadas. Así, en la colonia El Sol, $25 \%$ era trabajador por cuenta propia, $35 \%$, obrero y $30 \%$, empleado. En Santo Domingo $22.5 \%$ era trabajador por cuenta propia, $15 \%$, obrero y $37.5 \%$, empleado. 6

5 Estos porcentajes consideran solo los hogares en que el jefe es hombre ya que en $10 \%$ de los hogares de El. Sol y en $17.5 \%$ de los de Santo Domingo no hay hombre y el jefe es una mujer sola viuda, separada o soltera.

6 Estos porcentajes se refieren al total de hogares, es decir consideran también a los hogares $\sin$ hombre.
En cuanto al trabajo femenino, es importante apuntar que en $29.1 \%$ de los hogares arrimados hay una mujer que desempeña un trabajo remunerado, la mayoría de las veces como trabajadora por cuenta propia (comercio) o doméstica, mostrando así mayor participación en el mercado de trabajo que la que alquila o es propietaria de una vivienda. ${ }^{7}$ Es muy probable que esta situación se deba, justamente, a su condición de arrimada que le posibilita un apoyo doméstico (el cle las mujeres del otro hogar) del cual care. cen quienes no comparten.

Los ingresos en los hogares arrimados son levemente menores que los de inquilinos o propietarios en la misma colonia, salvo en los casos de propietarios que alquilaban cuartos. ${ }^{8}$ En El Sol, $50 \%$ de las familias declaró recibir entre uno y dos veces el salario mínimo y en Santo Domingo este ingreso representaba $57 \%$ del total. Sin embargo, estos ingresos menores se acrecientan dentro de la estructura del gasto mensual, en la medida en que su gasto en vivienda es mínimo o muy bajo. Así, $67.5 \%$ de las familias que comparten en El Sol y $77.5 \%$ de las de Santo Domingo declararon no tener gastos en vivienda (directamente) y 10000 pesos es el promedio men-

\footnotetext{
7 Efectivamente, en las colonias estudiadas se encontro que sólo $27.9 \%$ de las propitarias y $24.7 \%$ de las que alquilan desempeñan trabajo remunerado dentro o fuera del hogar. Véase Coulomb, Inquilinato, 1990.

${ }^{8} \mathrm{El}$ ingreso familiar promedio de las familias que comparten era (en 1989) de 435000 pesos mensuales. Los inquilinos ganaban en promedio 502 o00. los propietarios $545000 \mathrm{y}$ los propietarios arrendadores, 674000 . Véase Coulomb, Inquilinato, 1990 , apéndice.
} 
sual de las familias que sí reconocieron desembolso en este rubro. En cambio, los inquilinos de esas colonias declararon un gasto promedio mensual en el renglón vivienda de 50000 pesos y los propietarios de 16000 pesos. ${ }^{9}$

\section{c) Trayectoria residencial}

Una hipótesis importante planteada en relación con la vivienda compartida la identificaba con la primera opción habitacional de las parejas recién formadas, de los jóvenes y de los que apenas llegaban a la ciudad. En las colonias encuestadas esto es sólo parcialmente cierto.

En efecto, aparte de los antecedentes demográficos ya mencionados, en muchos casos (30\% del total de los encuestados) la familia encuestada tuvo una vivienda anterior y la ocupó como inquilino ( $45.8 \%$ ) o incluso como propietario (8\%). Además, el año promedio de formación de la pareja que comparte en la actualidad es 1976 y el año promedio de llegada a la casa encuestada es 1980 . Es decir, hay muchos casos en que los arrimados debieron tener una vivienda anterior en condiciones más independientes y el compartir fue una elección efectuada con posterioridad a ese intento.

\section{LA VIVIENDA COMPARTIDA. CONDICIONES DE VIDA DE LOS ARRIMADOS}

\section{a) Los servicios}

Tal como se dijo anteriormente, las colonias seleccionadas tienen casi 20

\footnotetext{
${ }^{9}$ Coulomb, Inquilinato, 1990.
}

años de formadas por lo que han incorporado, en general, la mayor parte de los servicios urbanos. Así, en El Sol $\mathbf{8 7 . 5 \%}$ de los arrimados dispone de electricidad oficial, $92 \%$ tiene agua (37.5\% dentro de la vivienda y el resto en el predio), y $95 \%$ tiene algún sistema de drenaje (62.5\% está conectado al alcantarillado público). En Santo Domingo, $97.5 \%$ tiene agua (47.5\% dentro de la vivienda), y 95\% tiene sistema de drenaje (42.5\% al alcantarillado).

\section{b) La vivienda}

La mayor parte de los arrimados (67.5\% en El Sol y 70\% en Santo Domingo) comparten el lote, en donde han edificado una vivienda para uso independiente de la de los propietarios. En general, esta vivienda ha sido construida por los propietarios (45\% en El Sol y 52.5\% en Santo Domingo) $\mathrm{y}$, en menor proporción (30\% en El Sol y 35\% en Santo Domingo), ha sido ejecutada por las dos familias en conjunto.

La vivienda de los arrimados consiste en uno (37.5\% en El sol y $40 \%$ en Santo Domingo) o dos cuartos (25\% en el Sol y 35\% en Santo Domingo) de uso exclusivo, dándose casos en que llegan a disponer de tres cuartos para ellos (10\% en El Sol y $7.5 \%$ en Santo Domingo). Las superficies totales varian, pero la mayoría dispone de 16 a $20 \mathrm{~m}^{2}$. El material más utilizado en muros es el tabicón ( $75 \%$ en El Sol y $92 \%$ en Santo Domingo) y en techos, la losa de concreto $(50 \%$ en El Sol y $65 \%$ en Santo Domingo). El piso puede ser 
de cemento pulido ( $52 \%$ en El Sol y $32 \%$ en Santo Domingo) o cemento rústico ( $35 \%$ y $62 \%$ respectivamente).

Al mismo tiempo, los arrimados disponen para ellos de otros recintos de uso específico tales como el baño (92.5\% en El Sol y 87.5\% en Santo Domingo) o la cocina ( $87.5 \%$ en ambas colonias) lo cual representa ventajas que no siempre tienen otros tipos de ocupantes como, por ejemplo, los inquilinos (en El Sol, sólo $56.3 \%$ de los inquilinos tiene recinto baño en su vivienda y $76 \%$ de ellos tiene recinto cocina).

Así también, ya sea porque comparten con el dueño o porque les pertenece, los arrimados disponen de bastante equipo doméstico. En El Sol, 92.5\% de las familias encuestadas tiene radio, $92.5 \%$, plancha, $87.5 \%$, televisor en blanco y negro, $55 \%$, tocadiscos o grabadora, $50 \%$, refrigerador y $42.5 \%$, lavadora eléctrica. Entre los inquilinos de esa colonia, estos porcentajes son $86.3 \%, 85 \%, 67.5 \%, 48.8 \%, 35 \%$ y $22.5 \%$, respectivamente. En Santo Domingo, $95 \%$ de los arrimados tiene radio, $92.5 \%$, plancha, $90 \%$, televisor en blanco y negro, $47.5 \%$, grabadora o tocadiscos, $40 \%$, refrigerador y $30 \%$, lavadora.

EL PROCESO DE COMPARTIR LA VIVIENDA. VENTAJAS Y DESVENTAIAS

Como se dijo previamente, el compartir la vivienda es un acuerdo solidario entre familias. Sin embargo, los arrimados asumen a veces ciertos compromisos económicos, no reconocidos como gasto en vivienda, ya sea en la forma de una contribución en dinero a los propietarios o mediante el pago de algunos servicios urbanos. Así, 51\% de los encuestados reconoció aportar una cuota mensual (en todos los casos inferior a 25000 pesos), $51 \%$ paga o participa con los dueños de la vivienda en el pago del agua, $66 \%$ en el de la luz y $54 \%$, en el del predial. Del mismo modo el $60 \%$ ayuda de alguna manera en las tareas cotidianas y domésticas de la familia principal, entre las cuales se encuentra la construcción y consolidación de la vivienda general.

Es probable que este tipo de acuerdo sea posible porque se trata, por lo general, de familiares muy cercanos. En el $62 \%$ de los casos, los arrimados son hijos de los poseedores de la vivienda y en $16.3 \%$, son hermanos. Del mismo modo, puede atribuirse a la cercanía de las relaciones existentes la estabilidadd de los arrimados en la vivienda pues, al contrario de lo que se pensaba, el compartir no es siempre una situación habitacional transitoria y de corto plazo; el tiempo promedio que han permanecido los arrimados en la vivienda encuestada es de 34 meses (42 meses en El Sol y 27 meses en Santo Domingo).

La permanencia de los arrimados compartiendo una vivienda confirma la opinión que ellos tienen de su propia situación. Así, 90\% declaró que prefería compartir vivienda y sólo $8.8 \%$ desearía comprar otra vivienda. Así también, $\mathbf{8 7 . 6 \%}$ de los encuestados encontró ventajas en la situación de compartir la vivienda, siendo la principal, de tipo económico y, en concreto, que permite ahorrar (40\%). Otras ventajas 
mencionadas son el apoyo doméstico entre familias (17.5\%) o el vivir con familia (7.5\%). Entre las desventajas de la situación se mencionó principalmente la falta de privacidad $e$ independencia (12.5\%), pero $53.8 \%$ de los encuestados no encontró ni mencionó desventajas.

En consecuencia, pareciera ser que el compartir la vivienda no es una situación demasiado conflictiva para los arrimados. De hecho, $5 \%$ declaró que no se iría de la casa que comparte por ningún motivo, $25 \%$ se iría sólo si surgieran conflictos con los dueños y $21 \%$ si encontrara un lugar mejor para habitar (pero $90 \%$ no había buscado otra vivienda). Además, $93 \%$ declaró no tener ningún problema con respecto a la familia principal.

\section{CONCLUSIONES}

Los antecedentes presentados hasta ahora permiten señalar algunas situaciones importantes las que, por lo reducido del estudio y de la muestra, no pueden ser consideradas como expresión propia de la situación general prevaleciente en relación con la vivienda compartida y sus ocupantes. Deben, más bien, ser concebidas como conclusiones preliminares o como hipótesis a comprobar con una profundización y ampliación de la ex. periencia empírica. Cabe sí, señalar, que este trabajo ha permitido elaborar una descripción inicial de la vivienda compartida y sus ocupantes, detectar algunas situaciones no esperadas y avanzar en la definición de las categorías que permitirían conceptualizar el objeto de estudio.

En primer lugar, parece evidente que el compartir la vivienda no es una fórmula transitoria de solución al problema habitacional utilizada por los jóvenes que inician una nueva familia sino que, cada vez más, representa un mecanismo normal para obtener vivienda, similar al alquiler o a la compra de un lugar para vivir. Es decir, el ser arrimado no parece ya como una situación de emergencia sino que se ha transformado o se está transformando en una condición residencial estable y natural para las familias, al menos, en los sectores populares urbanos.

Al mismo tiempo, esta forma de acceso a la vivienda no tiene tanto que ver con las condiciones económicas de la familia arrimada (dificultades para invertir o gastar en vivienda propia o alquilada) sino, más bien, con las limitaciones de la oferta de vivienda para sectores populares que caracteriza cada vez más a las grandes ciuda. des. La situación en relación con los sectores medios no fue investigada en esta oportunidad.

En segundo lugar, las condiciones de vida de los arrimados no parecen diferir mucho de los inquilinos o los propietarios de vivienda en colonias populares. Es decir, los arrimados no viven peor que sus vecinos y esto ha llevado a una aceptación de esta forma habitacional e, incluso, a reconocer ventajas en ella.

Por último, parece existir una disposición por parte de los propietarios a recibir a sus familiares cercanos (especialmente hijos) como arrimados en su vivienda (en la mayoría de los casos 
han sido los propietarios quienes han construido la vivienda para los arrimados). Esto podría significar que uno de los objetivos de obtener la propiedad y la regularización de las viviendas para los antiguos autoconstructores, es poder facilitar la permanencia de los hijos (o de algunos hijos) cerca de sus padres.

El compartir tendría entonces, también, una connotación cultural. Desde esta perspectiva, el "patrimonio" representaría tanto una propiedad a heredar de los padres a los hijos como una posibilidad de solucionarles, en vida, el problema habitacional cuando establecen su propia familia.

\section{BIBLIOGRAFÍA}

Coulomb, René, México: la politica habitacional en la crisis, CENV, México, 1990. , y otros, Inquilinato y vivienda compartida en América Latina, CENV, México, 1990.

Espinoza, V. y A. M. Icaza, "Hogares $y$ allegados en el área metropolitana de Santiago", SUR, Documento de Trabajo núm. 117, Santiago, 1991.

Gilbert, Alan, "Arrendatarios y autoconstrucción: selección y restricçiones en el mercado de vivienda en los países de menores ingresos", EURE, núms. 39-40, Santiago, 1987.

Rodriguez, A., "Hogares allegados y viviendas populares", SUR, núm. 13, Santiago, 1987.

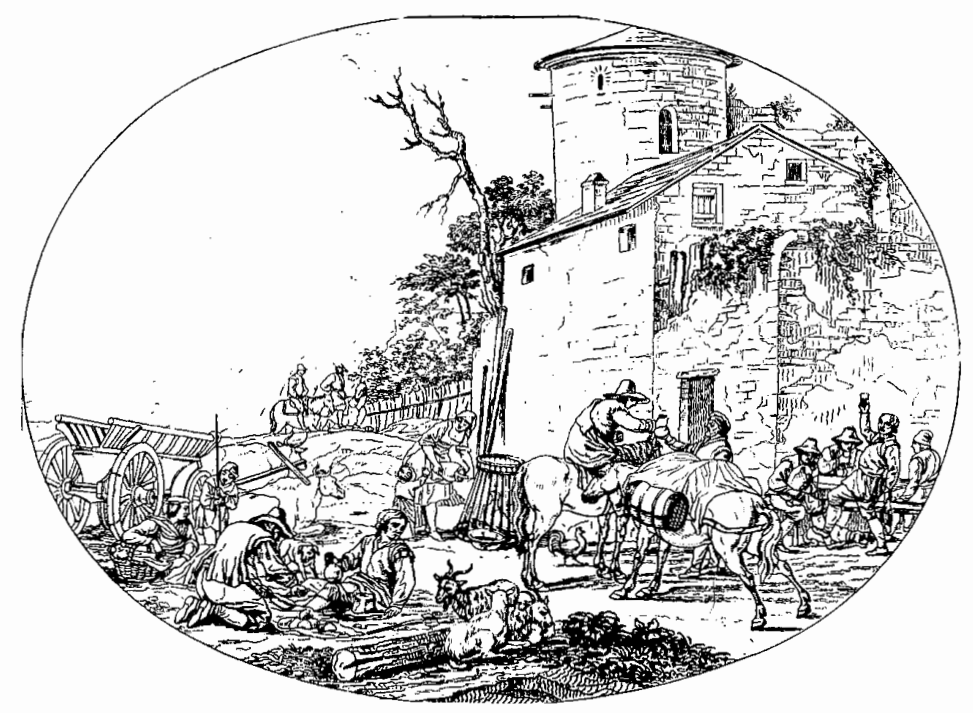

